\title{
Blood and urine levels of long half-life toxicants by nativity among immigrants to the United States
}

\author{
Peter Muennig a,*, Xiaoyu Song a , Devon C. Payne-Sturges ${ }^{\text {b, } 1}$, Gilbert C. Gee ${ }^{\mathrm{c}}$ \\ a Columbia University, Mailman School of Public Health, 600 West 168th Street, 6th Floor, New York, NY, 10032, USA \\ b United States Environmental Protection Agency, National Center for Environmental Research, Office of Research and Development, Ariel Rios Building MC 8723P, \\ 1200 Pennsylvania Ave. NW, Washington, DC 20460, USA \\ ' University of California, Los Angeles, School of Public Health, Department of Community Health Sciences, USA
}

\section{A R T I C L E I N F O}

\section{Article history:}

Received 26 July 2011

Received in revised form 23 September 2011

Accepted 28 September 2011

Available online 14 November 2011

\section{Keywords:}

Environmental toxicants

Immigrant and refugee health

Seemingly unrelated regression

\begin{abstract}
A B S T R A C T
One's place of birth is a major determinant of his or her exposure to environmental toxicants. By understanding biological burdens of long half-life toxicants by race and nativity we can better understand geographic variation in toxicant distribution. We used the National Health and Nutrition Examination Survey (1999-2006) biomonitoring data to examine differences in blood and urine levels of long half-life environmental toxicants of foreign-born relative to US-born people by race/ethnicity. We log transformed blood and urine measures of 51 environmental toxicants. We then used "seemingly unrelated regression," a robust technique for making multiple comparisons across a group of variables with correlated error terms, to examine differences in blood and urine toxicants by nativity and race. We found that, compared to native-born Americans, the foreign-born are generally more likely to be exposed to metals $(p<0.001)$ and organochlorine pesticides $(p<0.001)$, but less likely to be exposed to dioxin-like compounds $(\mathrm{p}<0.001)$ or polyflourinated compounds $(\mathrm{p}<0.001)$. While levels of toxicants varied greatly by region of birth, US-born participants had consistently higher levels of dioxin-like compounds and polyflourinated compounds.
\end{abstract}

(C) 2011 Elsevier B.V. All rights reserved.

\section{Introduction}

While some people in the world are exposed to few or no toxicants on a daily basis, others are likely exposed to thousands (Cranor, 2011; Woodruff et al., 2011). Some of these toxicants accumulate over the life course, so that the imprint of daily exposures early in life can remain with us even if we move to new geographic locations. This way, recent immigrants to the US can provide a global map of toxicant exposure (Eskenazi et al., 2011; Gordon et al., 2010; Miranda et al., 2011).

For example, recent immigrants to California who were born in Mexico tend to have much lower laboratory measures of polybromo diphenyl ethers (PDBEs) than Mexican-Americans born in California (Eskenazi et al., 2011). These compounds are used as flame retardants in textiles, plastics, wire insulation, and automobiles-products that are consumed much less frequently in poor nations.

There are many toxic compounds that fall under the category of PDBEs, and PDBEs are just one example of many broader categories of long half-life compounds that bioaccumulate in the body over years or decades. One challenge researchers face in making such comparisons is that there tend to be many compounds within a particular

\footnotetext{
* Corresponding author. Tel.: +1 347533 3415; fax: +1 2123423405 .

E-mail addresses: pm124@columbia.edu (P. Muennig),

payne-sturges.devon@epa.gov (D.C. Payne-Sturges), gilgee@ucla.edu (G.C. Gee).

1 Tel: + 1703347 8055; fax: + 17033478140 .
}

category of toxicants. For instance, consider organochlorine pesticides. Any given population group may have relatively higher biological levels of one type of organochlorine pesticide, but lower levels of another, providing a foggy picture of overall levels of this class of toxicant. Comparing biological levels of multiple compounds across two or more population groups can therefore be daunting.

In this paper, we use a novel methodological approach, seemingly unrelated regression (SUR) (Kling et al., 2007; Zellner, 1962). SUR provides an overall picture of a given group's levels of a toxicant category (such as PDBEs or organochlorine pesticides) by indicating whether, on average, laboratory levels across individual compounds within that category is higher for one group relative to another. In this paper, we investigate four classes of environmental contaminants-comprising 50 compounds in all. We then use SUR to describe variations in blood and urine levels within these four classes of toxicants by race, ethnicity, and nativity. The four toxicant categories are: 1) metals, 2) dioxins and polychlorinated biphenyl compounds (PCBs), 3 ) organochlorine pesticides and 4) polyflourinated compounds. These compounds were selected because they were the only long half-life compounds available to us in the National Health and Nutrition Examination Survey (NHANES) dataset we use in this study.

Most of these compounds have been linked to disease in humans (Ashford and Miller, 1992; Grandjean and Landrigan, 2006; Sharpe and Irvine, 2004; Vrijheid, 2000). For example, polychlorinated dibenzo p-dioxin and non-dioxin like PCBs act as a mixture of congeners that 
may damage endocrine or immunologic systems, and appear to cause intrauterine growth retardation in utero and congenital anomalies in newborns and children (CDC, 2009). Organochlorine pesticides have been linked to cancer (CDC, 2009).

Immigrants to the US appear to live much longer than native-born populations, and this is true across racial and ethnic categories (Fang et al., 1996; Muennig and Fahs, 2002; Singh and Miller, 2004; Singh and Siahpush, 2001). The reasons for this immigrant advantage are not fully understood, and toxic exposures could play some role. For instance, it is difficult to account for why rates of cancer are lower among immigrant groups who tend to be relatively heavy smokers than they are among native-born Americans SSingh, 2002 \#1170\}. Additionally, by examining long half-life toxicant levels among immigrant groups to the US, we can better understand the distribution of toxicants globally.

\section{Materials and methods}

Data for the study were drawn from the ongoing NHANES, a nationally representative sample of the US civilian non-institutionalized population (NCHS, 2011). As part of NHANES, the National Center for Health Statistics (NCHS) of the Centers for Disease Control and Prevention $(\mathrm{CDC})$ conducts biomonitoring of multiple environmental contaminants from a subset of survey participants. The NHANES employs a complex sampling design consisting of sampling at the county, household, and individual levels. Each year, specific sub-groups are oversampled including low-income whites in 2000, and blacks and Mexican Americans in other years. All analyses were adjusted to account for the complex sample design of the NHANES.

In the present analysis, several years of data (1999-2006) were merged to create a dataset containing 41,474 participants from 1 year of age onward. Subjects were included if their assessment contained interview, medical examination, and laboratory data. As some laboratory tests were only administered to a random sub-sample of the population, several outcomes have smaller overall sample sizes. Table 1 describes the final sample, including sample size by variable after excluding missing values. Sociodemographic information and medical histories of the survey participant and the family were collected during the household interview. Additional detailed information on the design and conduct of the NHANES survey as well as the publicly released data is available (NCHS, 2011).
In this secondary data analysis, we did not have access to data on specific countries of birth other than the US and Mexico in the public use data file (other countries are recoded by NHANES to prevent identification of subjects.). We therefore examined outcomes both by the available country of birth (US versus Mexico and other), by race (white, black, other), and ethnicity (Hispanic-Mexican/Hispanicnon Mexican/non-Hispanic). Although imperfect, this categorization provides a rough approximation of the region of origin of the majority of subjects. We assume that foreign-born Hispanic persons emigrate from Mexico, Latin American and the Caribbean, non-Hispanic white immigrants emigrate from Europe, non-Hispanic black immigrants emigrate from Africa, and most other immigrants emigrate from Oceana or from South, West, or East Asia. Approximately, 16\% of respondents in this latter category were born in the Philippines, $14 \%$ in China, $12 \%$ in Vietnam, $12 \%$ in India, and $10 \%$ in Korea (Census, 2010). The NHANES protocol was approved by a governmental institutional review board.

\subsection{SUR analyses}

We identified those compounds that were measured in the NHANES with a half-life of greater than 2-5 years using the National Report on Human Exposure to Environmental Chemicals (CDC, 2009). We grouped these long half-life compounds into four categories: metals (cobalt and cadmium), compounds related to dioxins and PCBs (39 putative toxicants), polyflourinated compounds (3 putative toxicants), and organochlorine pesticides ( 7 putative toxicants).

For each putative toxicant within each broader category of compounds, we log-transformed the dependent variable and conducted ordinary least squares regressions controlling for age and gender. The models were also stratified by nativity for each ethnic and racial group. Therefore, we created 51 separate regressions for each of the 4 racial and ethnic groups (along with all participants together) by nativity. This resulted in 510 ordinary least squares equations. For each class of compounds, we then applied seemingly unrelated regression (SUR). SUR provides joint significance testing across an entire category of outcomes (Zellner, 1962). Under the assumption that error terms are correlated across equations, SUR generates a variance-covariance matrix, which is then used to compute the standard error of the average impact. Thus, SUR inherently corrects for multiple comparisons within each category of compounds. The major disadvantage of SUR is that it

Table 1

Demographic profile of the analytic sample in the National Health and Nutrition Examination Survey, 1999-2006.

\begin{tabular}{|c|c|c|c|c|c|c|c|c|}
\hline & \multicolumn{4}{|c|}{ Native-born } & \multicolumn{4}{|c|}{ Foreign-born } \\
\hline & Hispanic & Non-Hispanic White & Non-Hispanic Black & Others & Hispanic & Non-Hispanic White & Non-Hispanic Black & Others \\
\hline \multicolumn{9}{|l|}{ Age distribution } \\
\hline $0-17$ & $71 \%$ & $33 \%$ & $55 \%$ & $67 \%$ & $23 \%$ & $15 \%$ & $29 \%$ & $18 \%$ \\
\hline $18-44$ & $17 \%$ & $28 \%$ & $24 \%$ & $22 \%$ & $47 \%$ & $37 \%$ & $43 \%$ & $44 \%$ \\
\hline $45-64$ & $7 \%$ & $18 \%$ & $12 \%$ & $7 \%$ & $18 \%$ & $17 \%$ & $20 \%$ & $25 \%$ \\
\hline $65+$ & $6 \%$ & $21 \%$ & $8 \%$ & $4 \%$ & $12 \%$ & $30 \%$ & $8 \%$ & $13 \%$ \\
\hline \multicolumn{9}{|l|}{ Gender } \\
\hline Male & $48 \%$ & $49 \%$ & $49 \%$ & $48 \%$ & $50 \%$ & $50 \%$ & $54 \%$ & $43 \%$ \\
\hline Female & $52 \%$ & $51 \%$ & $51 \%$ & $52 \%$ & $50 \%$ & $50 \%$ & $46 \%$ & $57 \%$ \\
\hline \multicolumn{9}{|l|}{ Marital status } \\
\hline Married & $29 \%$ & $50 \%$ & $22 \%$ & $28 \%$ & $52 \%$ & $55 \%$ & $37 \%$ & $60 \%$ \\
\hline Widowed & $4 \%$ & $11 \%$ & $7 \%$ & $4 \%$ & $5 \%$ & $16 \%$ & $4 \%$ & $6 \%$ \\
\hline Divorced & $5 \%$ & $8 \%$ & $8 \%$ & $7 \%$ & $3 \%$ & $5 \%$ & $7 \%$ & $4 \%$ \\
\hline Separated & $2 \%$ & $1 \%$ & $4 \%$ & $1 \%$ & $4 \%$ & $2 \%$ & $3 \%$ & $2 \%$ \\
\hline Never married & $55 \%$ & $25 \%$ & $53 \%$ & $53 \%$ & $29 \%$ & $20 \%$ & $43 \%$ & $26 \%$ \\
\hline Living with partner & $5 \%$ & $5 \%$ & $6 \%$ & $6 \%$ & $6 \%$ & $2 \%$ & $5 \%$ & $2 \%$ \\
\hline \multicolumn{9}{|l|}{ Annual family income } \\
\hline$<20,000$ & $35 \%$ & $22 \%$ & $40 \%$ & $32 \%$ & $45 \%$ & $28 \%$ & $31 \%$ & $25 \%$ \\
\hline$>20,000$ & $65 \%$ & $78 \%$ & $60 \%$ & $68 \%$ & $55 \%$ & $72 \%$ & $69 \%$ & $75 \%$ \\
\hline \multicolumn{9}{|l|}{ Adult education level } \\
\hline$<$ High school & $31 \%$ & $16 \%$ & $30 \%$ & $11 \%$ & $69 \%$ & $17 \%$ & $12 \%$ & $20 \%$ \\
\hline High school & $25 \%$ & $27 \%$ & $23 \%$ & $20 \%$ & $16 \%$ & $23 \%$ & $22 \%$ & $20 \%$ \\
\hline$>$ High school & $44 \%$ & $57 \%$ & $47 \%$ & $70 \%$ & $14 \%$ & $59 \%$ & $66 \%$ & $60 \%$ \\
\hline
\end{tabular}


does not yield an interpretable effect size; it only yields an overall pvalue across all comparisons.

We also explored whether lower rates of smoking, educational attainment, or income among some groups might account for any observed findings. These analyses help us to understand whether exogenous exposures (e.g., from occupations that might expose participants to toxicants) in the US might account for any observed differences in blood toxicant levels by nativity status. Analyses were limited to participants aged 12 years and older, and all biological samples were obtained from blood except for cobalt, which was obtained from urine.

Throughout this study, we assume that those with a lower geometric mean concentration of a given class of toxicant within the blood and urine have a lower level of exposure over their life course. The drawbacks of this assumption are discussed in detail in the Limitations section below.

\section{Theory}

Measured toxicant levels in adults can vary by nativity for various reasons. Exposures may vary by birthplace because the country of birth may have more stringent environmental protection laws, may have less industry, or may simply consume less commercial products. Exposures may also vary according to rates of smoking and socioeconomic status in the US (Muennig et al., 2010; Neidell, 2004). By holding adult sources of exposure in the US constant, we hope to glean insight into pre-immigration exposures to toxicants in foreignborn populations within the US. By employing SUR, we hope that we can get a sense of how variation in overall exposures to broad classes of toxicants, such as organochlorine pesticides affects the overall burden of such compounds.

\section{Results}

Table 1 shows the demographic profile of study sample in NHANES 1999-2006 by nativity. Children and adolescents make up the majority of Latino, non-Hispanic black, and "other" groups, but this is not the case with non-Hispanic whites. Males and females form a similar proportion of the total sample population in each group with the exception of "others," who are predominantly female. Participants born in Latin American have lower family income, and are less likely to be highly educated than three other immigrant groups. For instance, $45 \%$ of foreign-born Latin American adults have family income less 20,000, and $69 \%$ have not completed high school.

\subsection{Partially adjusted SUR models}

Table 2 shows the joint test $\mathrm{p}$ values of the levels of the compound categories stratified by nativity and race/ethnicity. The joint probability values represent the combined effects of all of the compounds within each category. Thus, if 10 compounds were analyzed, the joint test indicates whether, on average, the compounds could be found at a higher level in one group relative to another, and whether this difference was, in aggregate, statistically significant. The direction of the effect is indicated by an arrow. The p value for foreign-born groups with higher levels in aggregate relative to native-born groups is marked with an up arrow. The p value for foreign-born groups with a lower value in aggregate relative to native-born groups is marked with a down arrow. The data are presented in this simplified format because SUR does not afford an assessment of the magnitude of the effect other than the p-value. However, the online appendix shows the specific geometric mean values for each compound stratified by the group under study.

Across all racial/ethnic groups, our SUR models indicated that immigrants had significantly lower blood and urine levels of dioxin-like compounds and PCBs, and polyflourinated compounds (Table 2). In
Table 2

P-values of long-half life toxicants by nativity after adjusting for age and gender. All values are from blood samples except cobalt, which was obtained from a urine sample. The reference group is native-born in the same racial/ethnic category, so that an up arrow represents a higher value among the foreign-born ( $p$ value).

\begin{tabular}{lllll}
\hline $\begin{array}{l}\text { Foreign-born/ } \\
\text { native-born group }\end{array}$ & Metals $^{\mathrm{a}}$ & PCBs $^{\mathrm{b}}$ & $\begin{array}{l}\text { Organochlorine } \\
\text { pesticides }\end{array}$ & $\begin{array}{l}\text { Polyfluorinated } \\
\text { compounds }\end{array}$ \\
\hline All foreign-born & $\uparrow(<0.0001)$ & $\downarrow(<0.0001)$ & $\downarrow(<0.0001)$ & $\uparrow(<0.0001)$ \\
Latin American & $\uparrow(<0.0001)$ & $\downarrow(<0.0001)$ & $\downarrow(<0.0001)$ & $\uparrow(<0.0001)$ \\
Non-Hispanic White & $\uparrow(<0.0001)$ & $\downarrow(<0.0001)$ & $\downarrow(<0.0001)$ & $\uparrow(<0.0001)$ \\
Non-Hispanic Black & $\uparrow(<0.0001)$ & $\downarrow(<0.0001)$ & $\downarrow(<0.0001)$ & $\uparrow(<0.0001)$ \\
Others & $\uparrow(<0.0001)$ & $\downarrow(<0.0001)$ & $\downarrow(<0.0001)$ & $\uparrow(0.015)$ \\
\hline
\end{tabular}

Refer to Supplementary materials for the actual values.

a Includes cadmium and cobalt.

b Polychlorinated dibenzo p dioxin and non-dioxin like polychlorinated biphenyls.

contrast, immigrant groups tended to have higher levels of metals and organochlorine pesticides, although there was some variation by race/ethnicity.

Exploring overall concentrations of toxicants by class masks significant variation in individual compounds within each category of compounds by nativity and race. A few examples illustrate this variation. Overall levels PCBs and dioxins were lower among the foreign-born, yet, levels of 1,2,3,4,6,7,8-heptachlorodibenzo-p-dioxin $(34.2 \mathrm{pg} / \mathrm{g}$ for native-born, $39.3 \mathrm{pg} / \mathrm{g}$ for foreign-born; $\mathrm{p}<0.001$ ) were higher among in the combined foreign-born (see online appendix). While organochlorine pesticides were higher among the foreign-born in general, the pesticide Mirex was significantly higher among all of the native-born groups under study. Blood Endrin levels were marginally lower among Latin Americans $(p=0.1)$. While urine cobalt concentrations tended to be higher among most foreign-born groups, they were lower, but non-significantly so, among the "other" group-a grouping consisting primarily of Asians $(0.44 \mu \mathrm{g} / \mathrm{dl}$ versus $0.35 \mu \mathrm{g} / \mathrm{dl}$; $\mathrm{p}=0.13$ ).

\subsection{Complete SUR models}

The direction of the compound concentrations difference in immigrants and native-born Americans was similar for most of the putative toxicants before and after adjusting for covariates. The only exception was cadmium. In unadjusted analyses, cadmium levels were slightly higher among immigrants than among native-born Americans. After including adult education level, median cadmium levels were lower for foreign-born Americans than for native-born Americans.

\section{Discussion}

One of the challenges of understanding toxic burden borne by any given sociodemographic group is that it is quite difficult to interpret the variations in blood or urine levels of many different compounds. Within any class of toxicants, mean laboratory values can vary greatly from one compound to the next. When geometric mean blood and urine levels of some compounds within a given class are higher and others are lower, it is difficult to get a sense of whether the overall pattern of laboratory values differs significantly from one ethnic group to another. We show how SUR can serve as a useful tool for improving our understanding of a given group's overall laboratory levels of multiple compounds.

Using SUR, we find that there is substantial variation in measured laboratory levels of broader classes of compounds by nativity using nationally representative data from NHANES. Specifically, we find that foreign-born persons appear to have relatively higher blood 
and urine levels of metals and organochlorine pesticides, and lower levels of polyflourinated compounds and dioxins or PCB than U.S. born persons.

We also find little evidence that these laboratory values change when smoking, income, or educational attainment are added to the models. This suggests that differing smoking levels observed among the foreign-born do not account for the lower geometric mean levels of polyflourinated compounds and dioxins or PCBs seen among immigrant groups to the US. It also suggests that low socio-economic status as measured by education and income does not explain the observed higher levels of metal levels among the foreign-born relative to the native-born.

We found one exception to these trends. In models excluding education, cadmium levels were higher among the U.S. born. Yet, when controlling for education, the trend reversed so that cadmium levels were higher among immigrants. Blood levels of this compounded varied greatly by educational attainment, and prior literature shows that cadmium bioconcentrates in smokers (Lewis et al., 1972). This suggests that future studies of this compound should account for education as a potential confounder.

While we present blood and urine concentrations of a toxic class of chemical across many compounds at just one point in time, there is evidence that living in the US is a primary source of exposure, at least for PDBE. One cross-national and cross-generational study of Mexican immigrants, for instance, showed that children born to Mexican-American immigrants in California had PBDE levels that were three-fold higher than their mothers and seven-fold higher than children born in Mexico (Eskenazi et al., 2011). This study supports our finding that this and similar compounds tend to be elevated across all non-immigrant groups.

Our findings do not provide concrete support for the hypothesis that one's history of toxicant exposure is a contributor to the healthy immigrant effect. The geometric mean blood and urine concentrations of some compounds were lower among the foreign-born, but others were higher. The fact that blood and urine geographic mean concentrations of two of the four classes of compounds were lower among the foreign-born does not remove the possibility that lower toxic exposures across multiple compounds throughout life contribute to the healthy immigrant effect. In fact, it is possible that those compounds observed to be higher in the blood and urine among the foreign-born may have represented exposures that occurred here in the US, and not in the home country. For instance, organochlorine exposures tend to occur in agricultural work, and cadmium and cobalt exposures can occur in blue-collar industrial jobs that are now overrepresented by foreign-born workers.

There was significant variability across foreign-born groups with respect to the total levels of the classes of toxicants we study here. However, levels among the foreign-born tended to be consistently higher or lower than native-born people across racial and ethnic groups. This could just be by chance. Note the higher levels of cobalt among the foreign-born, for instance China is the top producer of cadmium with almost one-sixth of the world's share closely followed by South Korea and Japan (Brown et al., 2009). Wealthier European populations that eat shellfish are also at risk (CDC, 2009) and the Democratic Republic of Congo, in Central Africa, mines over $45 \%$ of the world's cobalt ore (Brown et al., 2009).

Our finding that all immigrant groups had lower levels of polyfluorinated compounds and dioxins or PCBs, on the other hand, was more in line with expectations. Polyfluorinated compounds have been industrially manufactured for over 50 years in developed countries for surface treatment in carpets, textiles, leather and paper, as well as in numerous other industrial and consumer applications (CDC, 2009). Dioxins and PCB compounds are by-products of various industrial processes and combustion activities, such as paper mills and incinerators. Even where stringent regulations now exist, weaker regulatory standards in the past can leave a long-term environmental footprint. Therefore, wealthy countries tend to have higher levels of these compounds in the environment. For dioxins and PCBs particularly, this effect may be multiplied by the higher consumption of meats, fish and dairy products in developed countries (Bilau et al., 2008). Foreign-born persons from rural areas, from countries less affected by globalized food chains, and from low-income families (who cannot afford to consume a good deal of meat and fish) might all be expected to have lower blood levels of these compounds.

Mirex and Endrine appear to be less prevalent in people born both in developing nations (which tend not to use chemically-treated materials) and in the European Union (which tends to have a more stringent regulatory environment). DDT is still widely used in several African and Asian countries, as the World Health Organization (WHO) recommends use of indoor spraying with DDT to battle malaria (Roberts et al., 2000). Not surprisingly, levels of these compounds are higher among the foreign-born.

\section{Limitations}

The safe levels of most of the compounds under study have not been firmly established, and compounds within a given category likely vary in toxicity. We could not account for such variation. Therefore, even where exposures are lower overall, it is possible that a few highly toxic compounds can produce a higher risk of disease.

More importantly, our data are cross-sectional and there are no variables available pertaining to the length of time any participant has spent in the US. Therefore, the blood or urine levels of these compounds may not accurately represent one's total exposure or risk over the life course. Likewise, we were unable to account for the health effects of chemicals not measured in the NHANES or of short half-life exposures.

Third, while metals and PBDEs tend to bioaccumulate, they do so in tissues other than blood. Blood and urine levels of these compounds will fluctuate with stress, caloric intake, and medication use, among other factors. Across a large population, higher geometric means may indicate higher lifelong exposures, but they could also be impacted by other factors associated with immigration itself, such as stress.

Fourth, it seems reasonable to hypothesize that social groups vary in their biomarker concentrations due in part to differential exposures. But an important alternative (and not necessarily mutually exclusive) hypothesis is that group variation in biomarker levels also reflects group differences in the ability to metabolize compounds that may arise from other origins, such as genomic characteristics, diet, and other unmeasured factors.

Finally, we are unable to disaggregate by specific country of origin or to provide finer grade resolution within the groups under study. This is particularly problematic when drawing inferences around the "other race" group.

\section{Conclusions}

We uncover some striking findings. First, there is a good deal of variation in blood and urine geometric mean levels of toxicants between racial and ethnic groups overall (independent of nativity). Second, there is a good deal of variation in blood and urine geometric mean levels of toxicants between the native-born and the foreign-born overall. Third, these differences notwithstanding, native/foreign differences are remarkably similar across all racial and ethnic groups under study. For instance, foreign-born non-Hispanic whites have relatively lower levels of dioxins and PCBs than native-born people; this trend also exists in Hispanics, non-Hispanic blacks, and others. This is seen despite the fact that there is huge variation in concentrations between, say, others (mostly Asians) and Hispanics. We hope that this study will fuel additional research into the biological effects of toxic exposures in humans, an understanding of how toxicants are distributed across the globe. 
Supplementary materials related to this article can be found online at doi:10.1016/j.scitotenv.2011.09.077.

\section{References}

Ashford N, Miller S. Chemical exposures: low levels and high stakes. Risk Anal 1992;12. Bilau M, Matthys C, Baeyens W, Bruckers L, Backer GD, Hond ED, et al. Dietary exposure to dioxin-like compounds in three age groups: results from the Flemish environment and health study. Chemosphere 2008;70:584-92.

Brown TJ, Hetherington LE, Hannis SD, Bide T, Benham AJ, Idoine NE, et al. British Geological Society. World mineral production: 2003-2007. Available online at: http:// www.bgs.ac.uk/downloads/start.cfm?id=1388.

CDC. Centers for Disease Control, Prevention. Fourth national report on human exposure to environmental chemicals. Available online at http://www.cdc.gov/exposurereport/. Accessed 8/31/2010.

Census. US Bureau of the Census. Census 2010. Available online at http://www.census. gov/. Accessed 6/22/2011.

Cranor CF. Legally poisoned: how the law puts us at risk from toxicants. Harvard Univ Pr; 2011.

Eskenazi B, Fenster L, Castorina R, Marks AR, Sjodin A, Rosas LG, et al. A comparison of PBDE serum concentrations in Mexican and Mexican-American children living in California. environmental health perspectives; 2011.

Fang J, Madhavan S, Alderman MH. The association between birthplace and mortality from cardiovascular causes among black and white residents of New York City. N Engl J Med 1996;335:1545-51.

Gordon L, Payne-Sturges D, Gee G. Environmental health disparities: select case studies related to Asian and Pacific Islander Americans. Environ Justice 2010;3:21-6.

Grandjean P, Landrigan P. Developmental neurotoxicity of industrial chemicals. Lancet 2006;368:2167-78.
Kling JR, Liebman JB, Katz LF. Experimental analysis of neighborhood effects. Econometrica 2007;75:83-119.

Lewis GP, Coughlin LL, Jusko WJ, Hartz S. Contribution of cigarette smoking to cadmium accumulation in man. Lancet 1972;1:291-2.

Miranda ML, Edwards S, Maxson PJ. Mercury levels in an urban pregnant population in Durham County, North Carolina. Int J Environ Res Public Health 2011;8:698-712.

Muennig P, Fahs MC. Health status and hospital utilization of recent immigrants to New York City. Prev Med 2002;35:225-31.

Muennig P, Fiscella K, Tancredi D, Franks P. The relative health burden of selected social and behavioral risk factors in the United States: implications for policy. Am J Public Health 2010;100:1758-64.

NCHS. National Center for Health Statistics. National Health and Nutrition Examination Survey, 1999-2006. Available online at: http://www.cdc.gov/nchs. Accessed 6/20/11.

Neidell MJ. Air pollution, health, and socio-economic status: the effect of outdoor air quality on childhood asthma. J Health Econ 2004;23:1209-36.

Roberts DR, Manguin S, Mouchet J. DDT house spraying and re-emerging malaria. Lancet 2000;356:330-2

Sharpe R, Irvine D. How strong is the evidence of a link between environmental chemicals and adverse effects on human reproductive health? Br Med J 2004;328:447.

Singh GK, Miller BA. Health, life expectancy, and mortality patterns among immigrant populations in the United States. Can J Public Health 2004;95:I14-21.

Singh GK, Siahpush M. All-cause and cause-specific mortality of immigrants and native born in the United States. Am J Public Health 2001;91:392-9.

Vrijheid M. Health effects of residence near hazardous waste landfill sites: a review of epidemiologic literature. Environ Health Perspect 2000;108:101.

Woodruff TJ, Burke TA, Zeise L. The need for better public health decisions on chemicals released into our environment. Health Aff 2011;30:957.

Zellner A. An efficient method of estimating seemingly unrelated regressions and tests for aggregation bias. J Am Stat Assoc 1962;57:348-68. 